\title{
The perils of public debate
}

$\mathbf{T}$ he recent legal and political battles over assisted feeding for Terri Schiavo, a severely brain-damaged woman, thrust the neurological terms 'persistent vegetative state' and 'minimally conscious' into everyday discourse. Amid the tangle of medical facts and misconceptions and the blurring of ethical and scientific questions, one theme stood out clearly: the misuse and misunderstanding of science. For science to effectively inform such debates, scientists and the public need to develop a more nuanced understanding of what science can and cannot be expected to contribute to public policy discussions.

The scientific perspective is only one piece of the puzzle, and scientists should recognize the legitimate role of ethical principles, including those based in religion, in public debates. "We can get very gummed up very fast if we fail to separate and identify the elements in these controversies that are matters of fact from those that are matters of principle," says University of Pennsylvania neuroscientist Martha Farah. Even if it were possible to prove beyond doubt that Terri Schiavo had no sense of awareness and no hope of recovery, that would not provide a single, correct answer for the ethical question of whether to remove her feeding tube. Similarly, no scientific argument levied on either side of highly politicized debates about human embryonic stem cell research will determine whether destruction of embryos for biomedical research is ethical. Science cannot answer such questions, and it cannot replace the moral values on which they depend.

However, most people do base their position on such issues in part on their understanding of the facts, particularly when they are asked to weigh two opposing principles. "What looks at first like a difference in ethical principles may really be a difference in understanding about the empirical facts," says Farah. Scientists have a special ability to assess scientific evidence, and we have a responsibility to disseminate this information in a way that allows the public to make informed decisions. In Terri Schiavo's case, much confusion centered on whether or not she was aware or had any chance for meaningful recovery. Yet the diagnosis that she was in a persistent vegetative state, without consciousness, was made with a higher degree of certainty than was probably apparent to much of the public.

Some of the responsibility for such misunderstandings lies with journalists, who have an incentive to stir up controversy to attract readers. Nicholas Schiff, a Cornell physician and neuroscientist who studies severely brain-damaged patients, notes that he and his colleague were unable to convince a New York Times editor to change a misleading headline about their research on minimally conscious patients before the story went to print. The headline ('New research suggests that many vegetative patients are more conscious than previously supposed-and might eventually be curable. A whole new way of thinking about pulling the plug') illustrates the confusion surrounding the facts of this case. Schiff and a small number of scientists have worked to clarify the boundary between persistent vegetative state and minimally conscious state, with a long-term goal of developing more sophisticated methods to evaluate patients in terms of their likelihood to recover or be misdiagnosed. Such confusion in the press undermines these researchers' efforts to reduce the uncertainties in treating patients with disorders of consciousness.

Even with the most careful reporting, of course, science cannot provide absolute certainty. However, scientists can often provide a good estimate of the uncertainty. We do not understand the full neural basis of consciousness, but patients like Schiavo, who have suffered a global block of blood supply to the brain, sustain an overwhelming and permanent loss of the corticothalamic networks that seem to be required for consciousness. Likewise, it is unclear whether adult stem cells will ultimately present as much potential for therapeutic use as embryonic cells, but most scientists agree that the uncertainty is still far too great to justify dismissing human embryonic stem cell research. We must take care to explain that uncertainties are inherent in science, to present them honestly and to help determine which ones are particularly relevant to ethical choices. Although we can rarely provide the decisive answer that the public may wish to hear, we can provide information about likelihoods and how we interpret them, which may help the public weigh the strengths and weaknesses of the scientific evidence used to support different viewpoints.

Frustration with the misuse and misunderstanding of science in public debate may tempt scientists to stop participating in these discussions, but — as the stem cell controversy illustrates-we cannot afford this luxury. Instead, we should improve our ability to communicate effectively with the public by recognizing the ethical issues involved and by listening to the questions that people want us to answer, rather than presuming we know best what the public needs to hear. We should avoid speaking in utilitarian terms or implicitly placing science above other forms of knowledge; either is likely to make the public tune us out. In addition to providing information about specific issues, we need to help the public understand the scientific process, including the role of empirical (versus anecdotal) evidence in resolving conflicting views. We might also establish direct contacts with the public through lectures, discussions or websites rather than relying on intermediaries such as journalists and politicians, who may get the science wrong or present it in a biased way.

Incentives to manipulate scientific findings for political ends will always exist, but scientists can and should make it much more difficult for opportunists to misuse their words and their work. We already have a great advantage in this regard: the respect of the public. People in the US consistently give scientists a high vote of confidence, second only to physicians in most years ${ }^{1}$. In contrast, few express confidence in politicians or the press. More thoughtful communication will be the key to maintaining this confidence and putting it to good use.

1. Davis, J.A., Smith, T.W. \& Marsden, P.V. General Social Surveys: 1972-2002 Cumulative Codebook (National Opinion Research Center at the University of Chicago, Chicago, Illinois, USA, 2003). 